\title{
Engaging High School Students in Cameroon with Exam Practice Quizzes via SMS and WhatsApp
}

\author{
Anthony Poon \\ Cornell University \\ New York, New York \\ atp65@cornell.edu
}

\author{
Sarah Giroux \\ Cornell University \\ Ithaca, New York \\ sh104@cornell.edu
}

\author{
Parfait Eloundou-Enyegue \\ Cornell University \\ Ithaca, New York \\ pme7@cornell.edu
}

\author{
François Guimbretière \\ Cornell University \\ Ithaca, New York \\ fvg3@cornell.edu
}

\author{
Nicola Dell \\ Cornell Tech \\ New York, New York \\ nixdell@cornell.edu
}

\begin{abstract}
We created a quiz-based intervention to help secondary school students in Cameroon with exam practice. We sent regularly-spaced, multiple-choice questions to students' own mobile devices and examined factors which influenced quiz participation. These quizzes were delivered via either SMS or WhatsApp per each student's preference. We conducted a 3week deployment with 546 students at 3 schools during their month of independent study prior to their graduating exam. We found that participation rates were heavily impacted by trust in the intervening organization and perceptions of personal security in the socio-technical environment. Parents also played a key gate-keeping role on students' digital activities. We describe how this role - along with different perceptions of smartphones versus basic phones - may manifest in lower participation rates among WhatsApp-based users as compared to SMS. Finally, we discuss design implications for future educational interventions that target students' personal cellphones outside of the classroom.
\end{abstract}

\section{CCS CONCEPTS}

- Human-centered computing $\rightarrow$ Empirical studies in ubiquitous and mobile computing; Mobile phones; • Applied computing $\rightarrow$ Computer-assisted instruction.

Permission to make digital or hard copies of all or part of this work for personal or classroom use is granted without fee provided that copies are not made or distributed for profit or commercial advantage and that copies bear this notice and the full citation on the first page. Copyrights for components of this work owned by others than the author(s) must be honored. Abstracting with credit is permitted. To copy otherwise, or republish, to post on servers or to redistribute to lists, requires prior specific permission and/or a fee. Request permissions from permissions@acm.org. CHI 2019, May 4-9, 2019, Glasgow, Scotland Uk

(c) 2019 Copyright held by the owner/author(s). Publication rights licensed to ACM.

ACM ISBN 978-1-4503-5970-2/19/05 ..\$15.00

https://doi.org/10.1145/3290605.3300712

\section{KEYWORDS}

cell phones, education, field study, SMS, MIM, ICTD, HCI4D

ACM Reference Format:

Anthony Poon, Sarah Giroux, Parfait Eloundou-Enyegue, François Guimbretière, and Nicola Dell. 2019. Engaging High School Students in Cameroon with Exam Practice Quizzes via SMS and WhatsApp. In CHI Conference on Human Factors in Computing Systems Proceedings (CHI 2019), May 4-9, 2019, Glasgow, Scotland Uk. ACM, New York, NY, USA, 13 pages. https://doi.org/10.1145/3290605.3300712

\section{INTRODUCTION}

Researchers in $\mathrm{HCI}$ have demonstrated a longstanding interest in how new technologies can be used to improve education. For example, recent work in US contexts has included interactive tabletops [28], intelligent tutoring systems [31], and virtual educational spaces [8]. Education is also the most prevalent domain in HCI for developing contexts (HCI4D) [10], which focuses on low-resource and marginalized communities in the Global South. Recent studies have addressed Massive Open Online Courses (MOOCs) [25], educational games [23], and informal education or training [32]. Our paper contributes to this body of work by creating and studying an intervention that uses either short message service (SMS) or WhatsApp to deliver exam practice quizzes to high-school Baccalaureate students in Cameroon.

For secondary school students in Cameroon, few occasions are more important than their qualifying exam. Students spend the entire last month of their final year in school preparing for their Baccalaureate. Passing it and scoring well marks a successful debut into adult and professional life, opening opportunities for employment and continued education. Despite the importance of the Baccalaureate, many are left to their own devices. Students perform this month of exam preparation mostly outside of the school itself. Some students hire tutors or engage in group study sessions where possible. However, many students find it challenging to 


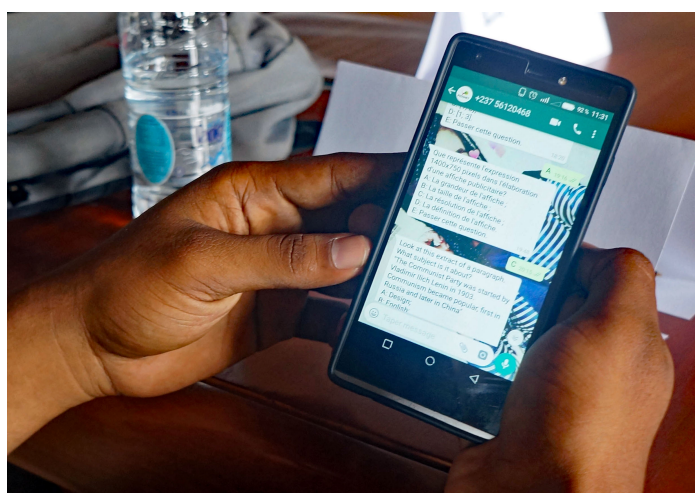

Figure 1: A student shows the quiz questions he has received and answered via WhatsApp on his phone.

source adequate study materials and other resources that will help them properly prepare for their exams.

Our goal was to explore the possibility of engaging highschool students with exam practice quizzes that were delivered directly to their personal phones via SMS or WhatsApp to improve studying. These quizzes were sent to students several times per week, and each consisted of 3 practice questions on multiple subjects. The questions were sent one at a time: Question 2 was sent only after the student responded to Question 1 . Thus, completing a quiz involved a multi-step, two-way interaction between the student and our automated system. We evaluated our intervention through a 3-week deployment with 546 students at 3 high schools in Cameroon, collecting quantitative system usage data and qualitative data from 9 focus groups conducted with 47 participants.

Our findings show that trust was a crucial decider of student participation. This trust included both students' perceptions of personal security in the socio-technical environment of SMS and WhatsApp, and trust in the specific organization communicating with them. In addition, we found that parents served as crucial gatekeepers to students' digital activities during the exam study period. Students, parents, and teachers did not widely view mobile phones as productive or studious devices. This view, combined with parental gate-keeping, impacted participation rates in our deployment, especially among WhatsApp participants. We discuss directions for future work and suggest changes to the presentation and deployment of future educational interventions that rely on personal cellphones outside of the classroom.

\section{RELATED WORK}

Our work intersects multiple sub-domains of $\mathrm{HCI}$ and builds on a rich body of prior work. Education is a frequent application domain in HCI4D and Information and Communication Technologies for Development (ICTD) literature [10] and the focus of learning technologies researchers. Our intervention uses SMS and WhatsApp. SMS has a history of diverse applications in ICTD [10], and there is growing research examining WhatsApp and other mobile instant messaging (MIM) platforms in educational settings [43]. Our work contributes to the space covered by these overlapping fields.

Education in HCI4D. Technology-based educational interventions in developing contexts have been studied in both formal and informal educational environments. In classrooms, researchers have created collaborative learning experiences by enabling students to play educational games on shared computers using multiple mice [34] and keyboards [45]. Games have also been deployed for education using other technologies, such as feature phones [15] and cameras [23].

MOOCs have also been applied to developing contexts for their promise of free education and increasing access for underprivileged students. Most MOOCs are created with a Western, educated, industrialized, rich, and democratic (WEIRD [18]) audience in mind, but some researchers have found that deploying MOOCs abroad can provide tangential benefits in the form of cultural and social capital [26]. However, the mismatch of assumptions between the course content and local context can be barriers to MOOC adoption [25], and it is uncommon for MOOC students to participate as an alternative to an unaffordable formal education [12]. Courses on mobile devices may help bridge this gap [14].

Outside of the classroom, a large body of work has focused on training and vocational education. These included peer-shared lessons on sustainable agricultural practices for farmers via voice messages [32] and locally-produced video training aids for community health workers (CHWs) [22, 29]. In 2016, Gill et al. [16] described a system of providing quizzes on a regular schedule via SMS for CHWs in Vietnam. These quizzes were provided over a 6-month period and CHWs were tested afterwards to determine if those who received quizzes performed better. Although the study did not find a significant performance difference, they reported that the low cost of SMS and the positive reception of the quizzes may make such interventions worthwhile [16].

A similar SMS-based quiz intervention was deployed to Uganda for patient health education. Danis et al. [9] sent multiple choice questions for HIV/AIDs awareness to factory workers over the course of a 2- to 3-week period. Patient health education has been the goal of several interventions from voice systems for Ebola awareness [47] to videos for discussing HIV/AIDS in culturally sensitive ways [41]. Researchers have also used MIM in South Africa to address educational and health-education needs [17].

SMS and MIM in Education. SMS's low cost and high availability have made it a frequent tool of both HCI4D interventions and educational interventions more broadly. SMS has 
been used within higher education classrooms to provide interactive activities and enable open channel feedback $[39,42]$. Other researchers used SMS to release educational information such as grades, enrollment, and announcements to university students $[6,38]$. Health education has also made use of SMS, with studies on sending messages on managing diabetes to parents of diabetic children [46].

Use of MIM tools, like WhatsApp, in educational domains is a growing research area as these tools become increasingly prevalent. Researchers have used MIM tools to provide students in higher-education courses with a channel for after-hours instructor support or to distribute additional educational materials [40]. WhatsApp and similar tools have been used to build educational games [7, 17], for class discussion in university settings [21, 24], and for sharing voice recordings for peer-feedback during language learning [44]

SMS in HCI4D. Automated SMS has a long history in HCI4D, with software libraries such as RapidSMS [36] being developed for the purpose of collecting data and building SMSbased interventions. SMS has been used as a means to manage and transfer mobile money, most notably M-PESA [19]. In the health space, a common use of SMS is for outpatient medication and appointment reminders [33]. Some researchers have used SMS to enable patients to ask health questions and seek advice or services [35]. SMS has also been applied as a medium for supervising health worker performance and providing performance feedback [11].

Our Contribution. Past work has focused predominantly on using either SMS or MIM for higher and adult education. We look at WhatsApp-based messaging in direct comparison to SMS within an intervention focused on high-school students in HCI4D contexts. Our use of graded quizzes for exam preparation is uncommon in educational messaging. And while past work has delved into the impact of MIM- or SMS-mediated interventions and how students interacted within them, we examine factors which influence students' ability or decision to participate in such interventions.

\section{RESEARCH CONTEXT}

Cameroon is a sub-Saharan nation with a population of approximately 23 million as of 2015. Due to its colonial history, education in Cameroon is distinguished by its two parallel systems based on the French and British models. In the French-speaking east and south regions of the country, encompassing the capital of Yaoundé where our study took place, the vast majority of students go through the francophone education system.

In both systems, primary education is 6 years long, compulsory, and free, although families are required to pay for uniforms and supplies. Secondary education is not compulsory or free, and costs vary widely as the majority of secondary schools are private institutions. Secondary education consists of 7 years of schooling, at the end of which, students take a qualifying exam. For students in the francophone system, this exam is the Baccalaureate. Passing this exam is crucial for entry into professional life or as part of an application for higher education. Students view the successful passing of the Baccalaureate as both an achievement and the beginning of adulthood. However, pass rates are historically low, with only $52.7 \%$ of students passing in 2016 [30].

Education levels in Cameroon have gradually improved, with $72.8 \%$ of the population completing primary school in 2014 as compared to $47.8 \%$ in 1990 . Enrollment has also increased in secondary schools [1]. However, schools remain underfunded and poorly equipped, corruption is endemic, educational materials can be expensive and in short supply, and security challenges have made education difficult or inaccessible in parts of the country $[1,13]$.

In the last decade, Cameroon has seen widespread adoption of cellphones and mobile devices. In urban contexts, such our study site of Yaoundé, cellphone adoption among youth is near universal. A recent national survey reported that $92 \%$ of respondents age 15-29 owned their own phone, up to $95 \%$ among urban youth [3]. These figures suggest that cellphones may provide an inexpensive means for delivering educational materials to students.

In this research, we worked with PICHNET, a locallybased NGO in Yaoundé with experience conducting surveys that maintains connections with local government affiliates. These affiliates helped us negotiate access to schools and teachers. Outside of PICHNET, members of our research team have worked in Cameroon for several years.

\section{INTERVENTION DESIGN}

In January 2018, we conducted informal meetings with educators in Cameroon and an exploratory focus group with 19 local secondary and university students. A lack of educational materials and the importance of the Baccalaureate on life after secondary school were frequent themes. We decided to focus on a mobile-based intervention that would deliver multiple-choice quizzes as test preparation materials directly to students' cellphones. From these discussions, in which most students described using WhatsApp as their primary means of mobile communication, we decided to deliver these quizzes via either SMS or WhatsApp.

We designed our intervention to increase student learning and better prepare them for the Baccalaureate in multiple ways. Foremost, our quiz was intended to invoke secondary learning effects by sending questions on a regular schedule to encourage students to practice at those times. Spaced or 


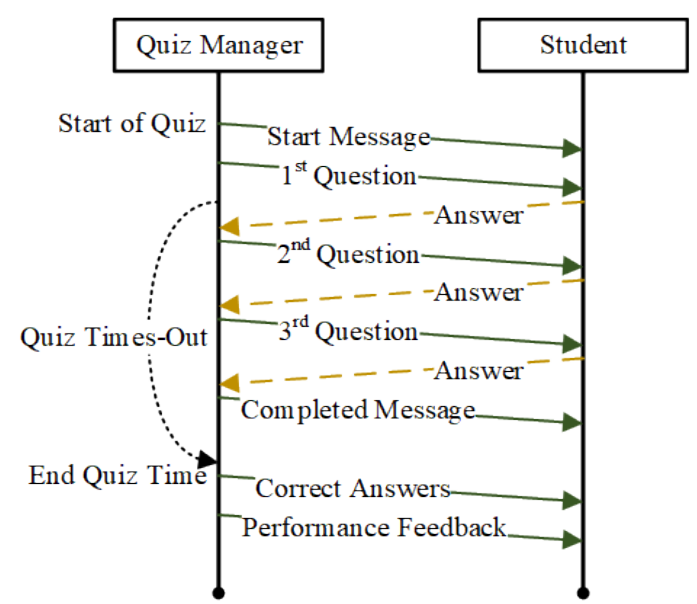

Figure 2: Typical multistage interaction of a student with a quiz. If the end time is reached before all questions are answered, the Manager skips to sending correct answers.

distributed practice can improve students' recall by reminding them of past learning episodes [4] and encouraging them to look up related materials via lateral learning [16]. As the quiz questions used material that teachers covered earlier in the year, students were challenged to retrieve information that they had previously learned. Such review improves retention and long-term learning $[2,37]$. Finally, the questions and answers provided by the intervention represent a new source of study materials for students.

As shown in Figure 2, our quizzes centered around a multistage interaction using a question and response model. As SMS and WhatsApp are asynchronous communication methods, it is difficult for the user to discuss more than one topic at a time. Thus, a new question was sent only after a prior question was answered. We grouped multiple questions into a unit of a quiz, which was automatically graded after some set time. This enabled us to have checkpoints for sending answers and performance feedback that was synchronized across all users and reduced the chance that students would share correct answers with each other. It also allowed us to remind students to study with regularly spaced messages even when the student did not answer any questions.

To simplify the handling of student responses, we decided to use multiple-choice questions with a standard of a single correct answer out of 4 answer options. We also included an fifth, explicit option for students to skip that question. Although this would count the question as being answered incorrectly, it was intended to encourage students to view more questions rather than to be stuck.

At the beginning of each quiz, students were sent a start message describing the number of questions in the quiz and brief instructions to respond with either the letter of the answer or "stop" to unsubscribe. This message was immediately followed by the first question. If the student answered all questions in the quiz, they were given a completion message stating that their results would be given to them soon.

We decided on 3 questions per quiz as a size that would be large enough to create meaningful groupings of questions but small enough that students could realistically complete all questions in the quiz period and avoid fatigue and disinterest. We chose to send 3 quizzes per week, on Monday, Wednesday, and Friday afternoons, again to balance quiz fatigue against regular interactions. The time of day was chosen based on our exploratory discussions, which revealed that students were typically less busy on weekday afternoons.

When each quiz ended, regardless of whether a student completed all 3 questions or not, students were sent a message with both the letter and a short textual explanation of each correct answer in the quiz. Students were then provided a feedback message that gave the number of questions answered correctly by the student in the past quiz, across all quizzes, and in the most recent 3 quizzes. We hoped that students would be motivated by feedback on their past performance, and that the longitudinal nature of this feedback would help them set and track goals of their own performance. At the end of the last quiz in the intervention, students received a message thanking them for their participation and wishing them luck on the Baccalaureate.

\section{Implementation}

From December 2017 to March 2018, we built a distributed, cloud-based workflow and integration service named PichnetMessagingManager. The purpose of this service was to manage the automated sending of messages, keep track of interactions with a large number of students, and manage state within those interactions. The Manager ran and stored student data on cloud platforms in Amazon Web Services. This allowed our application to easily scale up to handle more quizzes and be resilient to server failures.

We built two subsystems and integrations in PichnetMessagingManager to send and receive messages via either SMS or WhatsApp. For SMS, finding an acceptable third-party aggregator proved to be a challenge in Cameroon, particularly one that would allow our study participants to reply to our quizzes toll-free. Our implementation in Cameroon eventually relied on Mtarget, a France-based aggregator working on the Orange network. We made clear to participants at the start of the study that SMS replies were free.

We also had issues finding an acceptable provider for WhatsApp messaging. At the time of our implementation, WhatsApp did not officially support programmatic integration. We eventually settled on a third-party webservice that relied on automating the WhatsApp's web GUI to control a 
The severe legislation imposed by France in Cameroon during the mandate period is called:

A: Direct administration;

B: Permanent assimilation;

C: Provisional paternalism;

D: Code of the indiginate;

E: Skip this question.

Figure 3: An example quiz question used in the study translated from French. The correct answer is D, indiginate.

WhatsApp account on a separate internet-connected smartphone. We established an account using a local Cameroonian number with the PICHNET logo as the profile image to improve the chances students would trust our messages.

Quiz questions were written by 8 volunteer secondary school teachers in Yaoundé. These teachers provided multiple-choice questions in 7 subjects that appear on the Baccalaureate: English, French, geography, mathematics, philosophy, history, and information technology. An example question is shown in Figure 3.

Prior to deployment, the system was tested in the US and Cameroon throughout April 2018, and we did not encounter any lost or delayed messages via either SMS or WhatsApp.

\section{FIELD EVALUATION}

We evaluated our quiz intervention through a 3-week, 9-quiz deployment. All procedures were IRB-approved.

\section{Study Sites}

We chose 3 francophone schools for our study that represent the wide breadth of secondary educational institutions in Yaoundé. Lycée d'Elig-Essono is a government-run high school with approximately 3000 students and a typical class size of 70 to 80 students. Elig-Essono is large but also relatively affordable, charging an annual tuition of approximately 40,000 Central African Francs (XAF) (about \$71 USD) per year. Consequently, it is the most socioeconomically diverse of the schools in our study.

The second school, College Larousse is a privately-run institution. Larousse is the youngest school in our sample and also the smallest with approximately 1000 students. It has the smallest typical class size of 50 to 60 students and a tuition cost of approximately 140,000 XAF per year $(\$ 248$ USD). Larousse represents one of the many newer private schools that are attempting to expand their prestige.

The last school in our study is College Adventiste. This is an older, religiously-affiliated school with a student body of approximately 2000 and typical class sizes from 50 to 70 students. Annual tuition is about 160,000 XAF (\$283 USD). Due in part to its long history, Adventiste is one of the more prestigious secondary institutions in Yaoundé. At each school, we selected classes in the last two years of secondary schooling, equivalent to US $11^{\text {th }}$ and $12^{\text {th }}$ grade.

\section{Procedure}

We scheduled our intervention to be conducted alongside a larger survey of high school students at the end of April 2018. In this survey, we inquired about students' educational and employment aspirations, life outlook, trust in institutions, technology usage, and engagement in healthy versus risky behaviors. We worked with school administrators to set aside a few hours of time during normal class hours. Our staff gave a short presentation of the purpose of the study and recruited students to participate. Students completed the survey in the classroom and were each compensated 4,000 XAF (\$7 USD) for their time. As part of the survey, we obtained students' consent to participate in the quizzes, collected their mobile phone numbers, and asked if they preferred to be contacted via SMS or WhatsApp. In total, we recruited 546 students for our study: 450 via SMS and 96 via WhatsApp.

We started the quiz intervention at the beginning of May by sending an introductory message that tied the quizzes back to the survey that students took at the end of April. We also described the quiz, its purpose, and the quiz schedule. Quizzes were then sent for 3 weeks during May for a total of 9 quizzes. This schedule was chosen because students were released from school at the end of April and had a month of self-directed Baccalaureate preparation for the exam at the end of May. Thus, our quizzes coincided with the period during which students were busy studying for the test and ended right before the exam was administered.

During the deployment, we experienced challenges sending quizzes via WhatsApp. Although WhatsApp account numbers are typically the same as the user's mobile phone number, Cameroon had extended all local phone numbers from 8 to 9 digits in 2014 by prepending either a two or a six. However, the students who established their WhatsApp accounts prior to 2014 had the old, 8-digit version of their phone number as their WhatsApp account number. We resolved this issue by sending quizzes to both the 8- and 9-digit versions, but this resulted in WhatsApp participants starting a week later and receiving only 6 quizzes instead of 9 .

\section{Focus Groups}

At the end of the deployment, we invited students to focus group discussions to better understand their experiences in our study. We stratified students into three categories.

The first category included students who engaged more with the quizzes, defined as those who answered at least 1 question in each of the first 4 quizzes for SMS or the first 
quiz for WhatsApp. We conducted 3 groups with a total of 15 participants in this category (12 men and 3 women).

The second category included all other students who received quizzes. As study habits may differ between those with different levels of quiz engagement, we made this distinction between our categories to enable more diverse opinions to be expressed. We conducted 4 groups with 22 participants in this second category ( 7 men and 15 women).

Finally, our third category included students who consented to participate but were part of a non-intervention group that did not receive quizzes. Although they could not answer questions related to the quiz, we were still interested in their use of technology, SMS and WhatsApp, and views on institutional trustworthiness. We conducted 2 groups with 10 participants in the third category ( 3 men and 7 women).

In total, we conducted 9 focus groups with 47 participants (22 men and 25 women). Students were called on their given mobile numbers and recruited via voice. All focus groups took place in June and July 2018 after students had completed their exams but prior to receiving their results. 3 focus groups were held at the PICHNET offices, 1 in a researcher's home, and 5 in the private office of a local affiliate. These locations were all geographically central to Yaoundé.

For the first and second categories, we asked participants their opinions regarding the quizzes, reasons for responding or not, challenges or benefits experienced, suggestions for improvement, and more. Focus groups were held in French by a local, multilingual moderator, lasted between 50 to 80 minutes, and were audio recorded. We worked with the moderator to translate the questions from English and gauge their appropriateness.

\section{Data Collection and Analysis}

Our data consisted of quantitative data regarding students' interactions with the intervention and qualitative data from the focus groups. Focus group recordings were transcribed and translated by a local university student. We analyzed the data using thematic analysis [5], reading through the transcripts multiple times to identify codes. Examples of codes include Baccalaureate as Entry to Adulthood, Fear that Quiz was a Scam, and Parents Confiscated Mobile Phone. Codes were clustered into high-level themes that represent our main findings. Examples of themes include Importance of the Baccalaureate, Distrust of PICHNET, and Parental Control.

\section{FINDINGS}

\section{Understanding Participation}

Response and Participation Rates. Students provided in total 2678 parsable answers to quiz questions for an average of 297.6 responses per quiz. This was an mean response rate of $19.5 \%$, which was as high as $23 \%$ in the first 2 quizzes

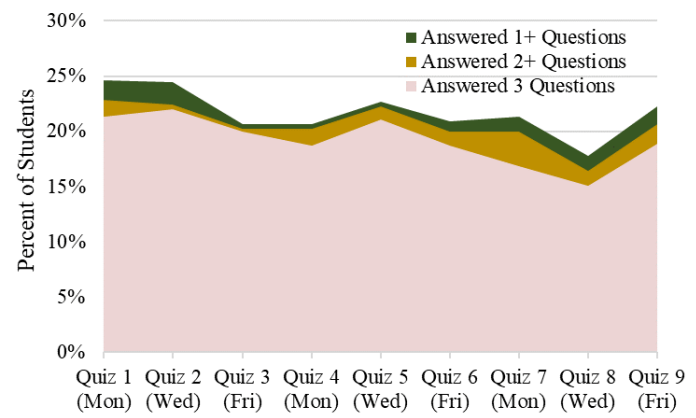

Figure 4: Student participation rate via SMS by percentage answering 1, 2, or 3 questions per quiz.

and declining as low as $15.6 \%$, but recovering right before exams were held. Given the novelty of the intervention in this context, we have no baseline for determining whether this is a high or low response rate. The shortage of exam study materials may suggest the rate is low. On the other hand, given the initiative's recentness and lack of a strong support program, one may also consider this rate high.

Participation rate refers to the portion of students who answered at least 1 question. As shown in Figure 4, participation was highest on the first quiz, where $24.7 \%$ of SMS-based users participated. The penultimate quiz saw the lowest participation rates, with $17.8 \%$ of SMS-based users. If a student chose to participate in a quiz, they tended to answer all three questions. For example, 96 students answered all 3 questions on the first quiz, whereas only 7 and 8 students answered 2 and 1 questions, respectively. This was likely heavily influenced by the structure of the quiz, in which the next question was sent immediately after a response was received for the previous question, encouraging continued interaction.

High and Low-Participators. 164 SMS-based users, or $36.4 \%$ of the SMS-based group, answered at least 1 question during the entire intervention. We call this subset of the students participators, and their participation can be characterized as bimodal. In Figure 5, participators are grouped by the number of quizzes in which they participated. While there was a set of high-participators, who answered a question in at least 7 quizzes, there were also many low-participators, who participated in 3 or fewer quizzes.

To better understand how high- and low-participators behaved across the duration of the intervention, we clustered all SMS participators into 2 groups using Ward's minimum variance method for hierarchical cluster analysis [20]. We then graphed the arithmetic mean of the number of questions answered per quiz within each cluster, as shown in Figure 6.

We see that high-participators $(n=87)$ stayed consistent throughout the intervention, answering questions on most quizzes at similar rates. Low-participators $(n=77)$ were 


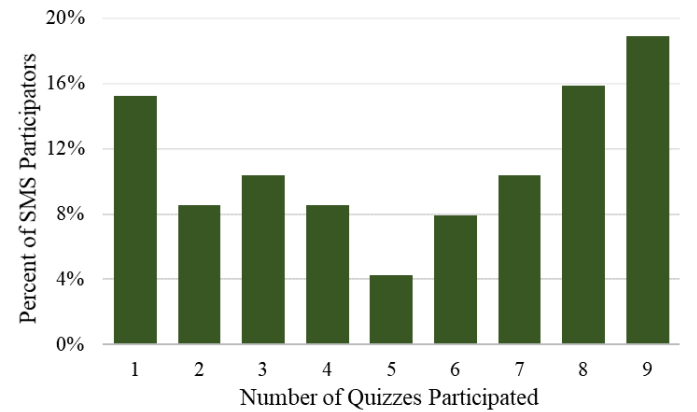

Figure 5: Percentage of SMS-based participators per number of unique quizzes they participated in, summing to $100 \%$.

more likely to answer a quiz question at the start of the intervention, but declined over time, stabilizing by the fourth quiz. This is likely due to novelty effect, with participation declining as interest in the new intervention decreased. It is encouraging that this novelty effect impacted only half of our participators. However, high-participators were likely intrinsically motivated to prepare for their Baccalaureate regardless of the prompting of our quiz intervention.

Unlike SMS-based users, the bimodal characterization is not apparent for WhatsApp-based participators, either due to the differences in timing or the smaller sample size of students who preferred WhatsApp.

\section{Trust As a Determinant of Participation}

To better understand students' motivations and incentives for answering quiz questions, we asked focus group participants why they chose to respond or not. Throughout the group discussions, many students described uncertainty and distrust as reasons for a lack of participation.

Confidence in PICHNET. This included distrust of PICHNET, the organization shown as sending the quizzes. The actions and inactions of the organization factored into students' trust calculation. For example, before the quiz intervention, students were asked to complete a survey with questions about their life goals and risky behaviors. The presentation of this survey is one example of an action that may have reduced trust in PICHNET, since many students were concerned about the sensitive nature of some survey questions. This could have led to people "opting-out" by refusing to respond to the quizzes.

On the other hand, actions such as being sensitive to the students' schedules and only sending questions during convenient times helped build trust and confidence. Some students told us how the quiz intervention itself built trust, as it was a tangible sign of PICHNET's intentions to help students.

Students emphasized the importance of the presentation of information about the quizzes to build confidence in the

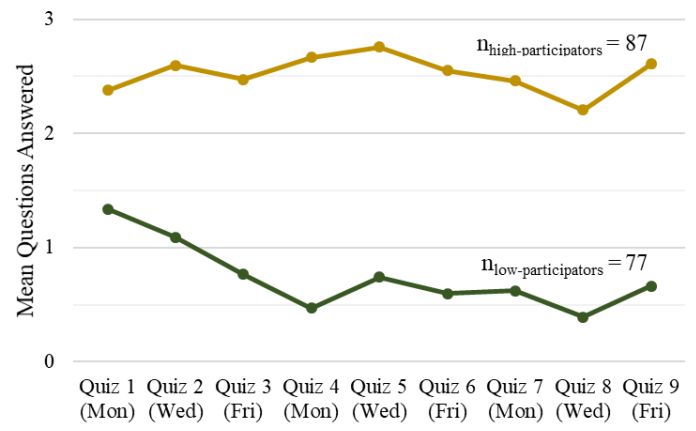

Figure 6: Average number of questions answered per quiz by high and low-participator, SMS-based students.

organization. Although our intervention began with an introductory message, many participants either missed reading this or did not pay it much attention. Instead, a common suggestion was to hold an in-person conference to explain our goals and introduce the quiz.

Insecurity in Environment. Regardless of confidence in PICHNET, students were also distrustful due to the uncertainty of the socio-technical environment in which they lived. Students were cautious answering quizzes because they were afraid of scams, harassment, and even had concerns about personal safety and kidnappings. Almost all focus group participants had heard stories of public corruption. Students reported receiving conflicting information about the Baccalaureate from multiple sources, and may have been afraid that the intervention was a prank or an attempt to cheat or harm them. This context of insecurity and uncertainty had a chilling effect on participation. As one participant described,

"It must be said that as exams approached, I received messages from everyone, whether my friends or classmates, saying that here are the questions that will come during the exam. As we do not know where to put our head anymore, we don't know what is true and what is wrong." [FGD9:C2:P46, Woman]

Some of our focus group participants admitted to being unaware of which organization was sending them quizzes, despite occasional reference to the PICHNET name. They did not make the connection between the quizzes and the organization that met them in their classroom with a survey. Unsure of the motivations of the sender, these students were reluctant to interact with an "unknown interest."

\section{Comparing SMS and WhatsApp Participation}

In the initial survey in April, 1117 students answered a question about their messaging service preferences for receiving communication. 400 students, $35.8 \%$, indicated that they 


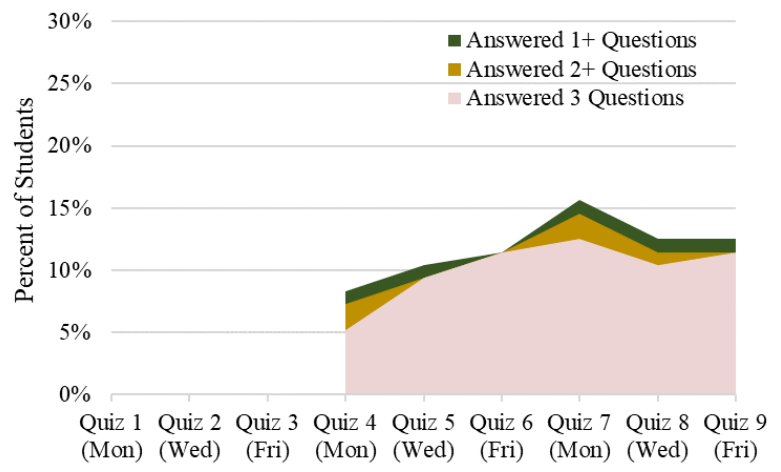

Figure 7: Participation rate via WhatsApp by portion answering 1, 2, or 3 questions per quiz.

would prefer to be contacted via WhatsApp with 206 preferring it exclusively to SMS or any alternative service. An additional 42 students did not prefer WhatsApp but provided a WhatsApp account number regardless. SMS remains by far the most common preference, with 863 students or $77.3 \%$ of respondents, 747 of whom preferred it exclusively. However, WhatsApp is clearly a popular alternative and has captured a large plurality of the student population.

Response and Participation Rates. Figure 7 shows the proportion of WhatsApp participants who provided parsable answers to 1,2, and 3 questions per quiz. Similar to SMS, WhatsApp participants tended to answer all 3 questions if they answered one. However as seen in Figure 8, they generally responded at lower rates than SMS participants.

Comparing WhatsApp and SMS participation is complicated by the fact that WhatsApp users only had the opportunity to participate in 6 quizzes. Comparing these WhatsApp quizzes to the last 6 SMS quizzes holds constant the chronological period and contents of the quizzes. Comparing to the first 6 SMS quizzes takes into account the user's experience receiving their first few quizzes (i.e., novelty effects).

We compared the sum of questions answered per student via WhatsApp against the same sums calculated for both the first-six and last-six SMS quizzes. Since the sample sizes of SMS and WhatsApp users is different $\left(\mathrm{N}_{\mathrm{SMS}}=450\right.$, $\mathrm{N}_{\text {WhatsApp }}$ =96) and the distribution of answer rates among students is non-normal, we use the Mann-Whitney U [27] test to perform these comparisons.

Participants who received quizzes via WhatsApp submitted an average of 1.96 responses, while those that received quizzes via SMS submitted an average of 3.84 responses during the first-six quizzes or 3.54 responses during the lastsix quizzes. Both differences were statistically significant $\left(\mathrm{U}_{\text {first-six }}=24522, \mathrm{p}_{\text {first-six }}=0.012, \mathrm{U}_{\text {last-six }}=23876\right.$, plast-six $=$

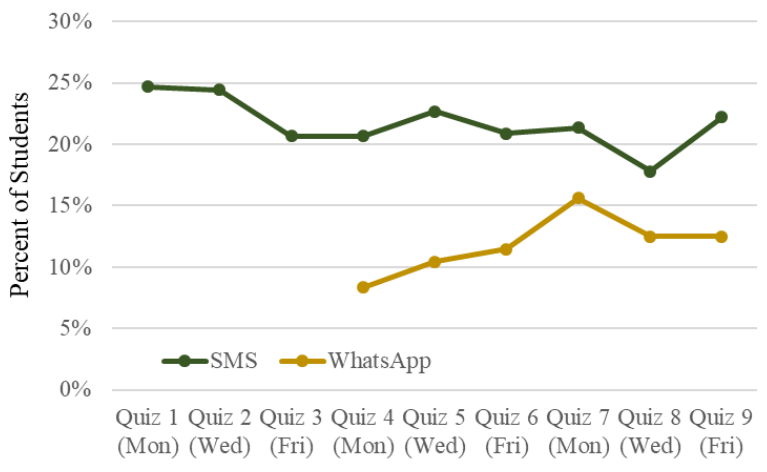

Figure 8: Comparing participation rates between SMS and WhatsApp for those answering at least 1 question per quiz.

0.045). This finding suggests that WhatsApp-based participants respond to fewer questions during the quiz intervention than SMS-based participants.

Students using WhatsApp answered at least 1 question on an average of 0.71 quizzes, while those using SMS did so on an average of 1.34 of the first-six or 1.26 of the lastsix quizzes. Both differences were statistically significant $\left(\mathrm{U}_{\text {first-six }}=24506, \mathrm{p}_{\text {first-six }}=0.013, \mathrm{U}_{\text {last-six }}=23898\right.$, plast-six $=$ 0.043). Given that WhatsApp participants, like SMS, were likely to answer all 3 questions in a quiz, this result suggests that WhatsApp participants' lower response rate may be due to less-frequent, rather than less-intense, participation.

This difference in participation was unexpected, as WhatsApp users are likely on average more comfortable with technology than SMS-based students. At a minimum, WhatsApp requires a smartphone and an Internet connection. Thus, WhatsApp users may be more receptive to an ICTbased educational intervention, and one might expect equal or higher participation rates as compared to SMS. We now use qualitative data from our focus group discussions to examine reasons for why this was not the case and in-fact saw lower participation among students using WhatsApp.

Normative Role of Cellular Technology. A few participants described how, in Cameroon, devices such as cellphones and messaging technologies were typically not seen as having productive or educational uses. While some students may use their phones for studying, the view that phones could be or were studious devices was far from universal. A few students in the focus group discussion noted this distinction and disconnect between the norms around their cellphone and our attempt to use it for education. Although this repurposing was not necessarily frowned upon by students, they did note we should do more to fight mismatched expectations from teachers and parents. As one participant said, 
"SMS most of the time is used to entertain. When you want to do something serious by SMS, it is possible, but you must add something specific." [FGD9:C2:P45, Woman]

Controls and sanctions exist to separate productive and educational environments from cellphones. For example, students attending Lycée d'Elig-Essono, the largest school in our study, reported that they were not allowed to bring their phones to campus. 11 of 36 participants across all of our quiz-receiving focus groups reported that their parents confiscated their phones during the month of study prior to the Baccalaureate.

Parental Control. Our participants still resided in their parents' homes and were subject to parental control and de facto guardianship. Students reported several ways in which parents exercised control over students' activities, especially during the study period. For example, in the physical space, parents restricted where students could go and who they could interact with using surveillance and curfews. While recruiting for focus groups, we spoke to parents multiple times about the purpose of our research and the organization. In 3 instances, parents even showed up to the focus groups in person to question us. One student highlighted the importance of the role parents play:

"You have to talk to the parents first because they are the ones responsible for their children, and they think that there are a lot of dangers out there, like witchcraft for example." [FGD3:C2:P12, Woman]

We found that parents played a crucial gate-keeping role in mediating interactions between a student and actors such as NGOs. This role was not necessarily unilaterally imposed by parents; it may also have been voluntarily requested by students. For example, students often gave the phone number of their parents as contact information instead of their own, thus allowing messages to be filtered through their parents. Parents used methods such as monitoring, acting as a digital intermediary, and confiscating phones to exert control over students' digital activities.

Smartphones and WhatsApp Disproportionately Impacted. As WhatsApp is only supported on smartphones, from our survey of student messaging preferences, we see that at least $39.6 \%$ of students in our sample own or have access to a smartphone for personal messaging. This reflects both the increasing adoption of smart devices and in particular among the vanguard group of secondary students, whose families pay for their education and thus are likely to come from middle to high socioeconomic backgrounds.

However, smartphones and WhatsApp may be disproportionately impacted by parental control. Among the students in our focus groups that reported their phones confiscated, 4 of these students in 4 groups explicitly reported that it was a smartphone but their parents also allowed the student to swap their SIM to a basic phone. Such a student would have been able to respond to SMS quizzes, but would not receive WhatsApp messages until their smartphone was returned to them. One participant described,

"The fact that students prefer SMS over WhatsApp is due to the fact that the parents deprive the children of optional telephones that include, for example, WhatsApp, Facebook, so that they do not move away from the real world. They are given simple phones to communicate. This is why the only means of communication they have are either calls or sending SMS." [FGD8:C1:P43, Man]

Differential parental attitudes towards smartphones provides one possible explanation for the significantly lower WhatsApp-based participation rates that we observed. Another student made this connection explicitly, identifying confiscation of WhatsApp-enabled phones as a reason why some would not have received the quiz and responded to it.

"It is because some students do not have phones.

It can also be due to the fact that people may have given WhatsApp numbers, but afterwards [their parents] took their WhatsApp and held it." [FGD3:C2:P14, Man]

\section{Role of Quizzes as a Study Aid}

To understand how the quizzes impacted students' exam preparation, we asked focus group participants to describe how the quizzes were helpful or harmful as a study aid.

Quiz Prompted or Challenged Students to Study. Several students in different focus group categories described the quizzes as prompting them to research a topic in greater detail or review past materials to correctly answer a question. Due to the difficulty of the questions and the regularity at which students received them, the quizzes were seen as challenging the respondent to do more studying than they would have otherwise. Some respondents noted that the month of independent study was a time in which quizzes were particularly valuable for inducing students to think and study. One participant said,

"The different quizzes ... permit me to try to revise the lessons that I may have forgotten. Through these quizzes I realized that I did not understand well, I still have to do some research." [FGD8:C1:P40, Man]

Quizzes Served as Focus of Collaborative Study. Students also used the quizzes as a focus for studying collaboratively with friends and family. One respondent sought help from an older siblings to answer the questions. Another shared the 
questions with a younger sibling who was about to take a lower-level exam. Multiple participants described sharing the quiz questions with friends who did not receive them. Some worked on questions together, while others discussed answers after the fact. One participant said,

"I discussed it with friends, but unfortunately, they did not receive the quizzes, so we often solved the questions together." [FGD7:C2:P36, Man]

Quiz Content Did Not Match Students'Needs. Although many students added the quizzes to their study routine, some students mentioned that the quizzes were not useful because the questions were not always relevant. Unlike standardized exams in the US, students in Cameroon study on different tracks with varying emphasis on different subjects. Although the quiz questions were written by local instructors, they did not fit the needs of all students equally. Questions may have been to hard, too easy, or irrelevant to their specialization. This lack of applicability was discouraging and may have led students to dismiss the quiz intervention in entirety, leading to lower participation.

Mismatched Expectations with Automated Interaction. Students were unfamiliar interacting with an automated messaging system. Participants were accustomed to sending messages to other humans and expected the quiz system to be similarly operated by a human. The fact that it was not ran counter to users' expectations. We received several messages thanking the operator or asking clarifying questions. Automated responses stating that the user's message could not be parsed then confused these students. A few users also sent messages that showed they were frustrated with our system's inability to understand their complaints.

\section{DISCUSSION}

Design Lessons for Trust. To improve participation, interventionists should emphasize building rapport with potential users. It is important to structure and present interventions to take trust in the organization and perceptions of security in the socio-technical environment into account. In the case of our quiz intervention, providing the introduction to the quiz via SMS or WhatsApp messages was too late to build trust with some of our students. Future work that examines students' conceptions of trust could provide additional insights into these issues. For example, we could focus on better understanding the models of trust that impact student and parent behavior, examine the differences in trust between high- and low-participators, or broaden the research to examine trust and insecurity in Cameroon over time.

As we found that parents play a central gate-keeping role for their children, we recommend that educational interventions properly communicate their goals and intentions to both parents and students, and obtain parental buy-in for student participation. Where meeting directly with parents is not possible, contacting parents and providing them with a method to query the intervention may be an acceptable alternative. For example, future iterations of our quiz intervention could send information home with students on paper pamphlets and provide parents with a phone number to call for questions. Parents that are aware of and approve of an education intervention can additionally encourage students to participate in those programs.

Without parental buy-in, parents' ability to place control on students' digital activities can reduce participation. We see how this may have resulted in lower answer rates in our quiz among WhatsApp-based students. Though SMS and WhatsApp are both messaging services, the latter is not a drop-in replacement for the former. WhatsApp, and similar MIM services, must confront additional challenges beyond higher cost to the user in the form of more expensive phones and service. These challenges arise because the perception of this technology as valid for non-entertainment purposes is not universal in our context.

Interventions that repurpose a technology with preexisting norms around its use must address those norms, and subsequent societal controls, in the intervention design. One way to address this may be to ask key actors to allow exceptions, such as convincing parents to allow smartphone use during certain times for study. However, cellphones as a non-educational device is a legitimate view, and designers must take care that encouraging exceptions to these rules could induce unintentional and negative repercussions.

Limitations and Improvements. Our findings suggest several improvements within the quiz interaction that could increase participation and its effectiveness. For example, customizing quiz questions to make them more relevant to individual students, changing the timing and duration of the quiz during the school year, and adjusting the workflow to provide students with answers to questions more quickly are all features that came up in our data. We plan to incorporate these changes into the redesign of our intervention before our next deployment. In addition, since participants discussed the social nature of studying for their exams, we plan to build better collaborative features into the intervention and evaluate whether they increase participation or promote learning. Such features may include group quizzes, collaborative and competitive feedback, and mechanisms to encourage students to discuss study materials with each other.

One limitation of our study is that we do not know the quizzes' impact on students' learning outcomes. A future iteration of this work should measure Baccalaureate pass rates among students receiving quizzes and compare them to a control group. Another limitation is that we did not directly 
examine parent and teacher views on smartphones and WhatsApp. We cannot conclusively state whether norms around smartphones versus basic phones drove behavioral differences in our intervention, or whether this may be caused by attitudes towards WhatsApp versus SMS. A closer examination of perceptions of MIM platforms may better expose the relationships between these technologies and acceptable uses in this context.

Future work could examine differences in participation rates and the degree of parental control based on demographic indicators. For example, gender biases in education or differences in socioeconomic status may impact participation rates. Such work may provide tools for future researchers to use demographic indicators to identify potential contextual pitfalls for a broad range of interventions.

\section{CONCLUSION}

This paper describes an intervention that sends regularlyspaced exam practice questions via SMS or WhatsApp to high school students' mobile devices in Cameroon. Our study shows that trust is a crucial decider of participation, including perceptions of personal security and trust in the organization communicating with students. We show how students and parents did not universally view mobile phones as productive or studious devices which can negatively impact participation when combined with parental control of students' digital activities. We discuss the implications of our work and ideas for the design of future educational interventions that target students' personal phones.

\section{ACKNOWLEDGEMENTS}

This research was funded by the US Department of Defense (W911NF-17-1-0029). We thank the PICHNET staff and local affiliates, in particular Vincent de Paul Onguene, Fidelice Mvogo Ebanda, Danielle Sandrine Mbele, Leopold Nikoul, Oscar Ayi, Pwe Hillary Bama, Sanama Alehonse Alarm, Serge Bahoken, and others, for their valuable contributions. This work would not been possible without them.

\section{REFERENCES}

[1] 2018. Education Fact Sheet: Cameroon. Technical Report. HALI Access Network. http://haliaccess.org/wp-content/uploads/2018/05/ Cameroon-Education-Facts-Sheet.pdf

[2] Olusola O. Adesope, Dominic A. Trevisan, and Narayankripa Sundararajan. 2017. Rethinking the Use of Tests: A Meta-Analysis of Practice Testing. Review of Educational Research 87, 3 (2017), 659-701. https://doi.org/10.3102/0034654316689306

[3] Afrobarometer. 2015. Cameroon. http://www.afrobarometer.org/ countries/cameroon-0

[4] Aaron S Benjamin and Jonathan Tullis. 2011. What makes distributed practice effective? Cognitive Psychology 61, 3 (2011), 228-247. https: //doi.org/10.1016/j.cogpsych.2010.05.004.What

[5] Virginia Braun and Victoria Clarke. 2006. Using thematic analysis in psychology. Qualitative Research in Psychology 3 (2006), 77-101. https://doi.org/10.1191/1478088706qp063oa arXiv:arXiv:1011.1669v3

[6] Paul Brett. 2011. Students' experiences and engagement with SMS for learning in higher education. Innovations in Education and Teaching International 48, 2 (2011), 137-147. https://doi.org/10.1080/14703297. 2011.564008

[7] Laurie Butgereit, Barend Leonard, Christine L E Roux, Himal Rama, Michele D E Sousa, and Thayendran Naidoo. 2010. Dr Math gets MUDDY: The "Dirt" on How to Attract Teenagers to Mathematics and Science by Using Multi-User Dungeon Games over Mxit on Cell Phones. IST-Africa 2010 Conference Proceedings (2010), 9. http://hdl. handle.net/10204/4085

[8] Alan Cheng, Lei Yang, and Erik Andersen. 2017. Teaching Language and Culture with a Virtual Reality Game. In Proceedings of the $2017 \mathrm{CHI}$ Conference on Human Factors in Computing Systems - CHI '17. ACM Press, New York, New York, USA, 541-549. https://doi.org/10.1145/ 3025453.3025857

[9] Catalina M Danis, Jason B Ellis, Wendy a Kellogg, Hajo Van Beijma, Bas Hoefman, Steven D Daniels, and Jan-willem Loggers. 2010. Mobile Phones for Health Education in the Developing World : SMS as a User Interface. ACM Dev'10: 1st Annual Symposium on Computing for Development (2010). https://doi.org/10.1145/1926180.1926197

[10] Nicola Dell and Neha Kumar. 2016. The Ins and Outs of HCI for Development. Proceedings of the 2016 CHI Conference on Human Factors in Computing Systems - CHI '16 (2016), 2220-2232. https://doi.org/10. $1145 / 2858036.2858081$

[11] Brian DeRenzi, Benjamin Birnbaum, Leah Findlater, Joachim Mangilima, Jonathan Payne, Tapan Parikh, Gaetano Borriello, and Neal Lesh. 2012. Improving community health worker performance through automated SMS. Proceedings of the Fifth International Conference on Information and Communication Technologies and Development (2012), 25-34. https://doi.org/10.1145/2160673.2160677

[12] Tawanna Dillahunt, Zengguang Wang, and Stephanie D. Teasley. 2014. Democratizing higher education: Exploring MOOC use among those who cannot afford a formal education. International Review of Research in Open and Distance Learning 15, 5 (2014), 177-196. https://doi.org/ 10.19173/irrodl.v15i5.1841

[13] Richard A Doffonsou, Christiane Bollo-tema, Joseph N Guessan Kouassi, and Uloma U Nwamarah. 2015. Cameroon: Joint 2015-2020 Country Strategy Paper and Country Portfolio Performance Review Report. June (2015).

[14] Eneza Education. 2017. Eneza Education. http://enezaeducation.com/

[15] Vanessa Frias-Martinez, Jesus Virseda, and Aldo Gomero. 2012. Mobilizing education: Evaluation of a Mobile Learning Tool in a Low-Income School. Proceedings of the 14th international conference on Humancomputer interaction with mobile devices and services - MobileHCI '12 (2012), 441. https://doi.org/10.1145/2371574.2371642

[16] Christopher J. Gill, Bao Le Ngoc, Nafisa Halim, Ha Nguyen Viet, Anna Larson Williams, Nguyen Van Tan, Marion McNabb, Lien Tran Thi Ngoc, Ariel Falconer, Hai An Phan Ha, Julia Rohr, Hai Hoang, James Michiel, Tam Nguyen Thi Thanh, Liat Bird, Hoang Pham Vu, Mahlet Yeshitla, Ha Van Nhu, and Lora Sabin. 2016. The mCME Project: A Randomized controlled trial of an SMS-Based continuing medical education intervention for improving medical knowledge among Vietnamese community based physicians' assistants. PLOS ONE 11, 11 (2016), 1-19. https://doi.org/10.1371/journal.pone.0166293

[17] Jean Greyling, Melisa Koorsse, Tawanda Ngundu, and Michael Kyazze. 2013. Mobile Instant Messaging (MIM) Applications to Assist Learning in South Africa. Educational Research for Social Change 2, 1 (2013), $30-47$.

[18] Joseph Henrich, Steven J. Heine, and Ara Norenzayan. 2010. The weirdest people in the world? Behavioral and Brain Sciences 33, 2-3 (jun 2010), 61-83. https://doi.org/10.1017/S0140525X0999152X 
[19] Nick Hughes and Susie Lonie. 2007. M-PESA: Mobile Money for the "Unbanked" Turning Cellphones into 24-Hour Tellers in Kenya. Innovations: Technology, Governance, Globalization 2, 1-2 (2007), 63-81. https://doi.org/10.1162/itgg.2007.2.1-2.63

[20] Joe H. Ward Jr. 1963. Hierarchical Grouping to Optimize an Objective Function. F. Amer. Statist. Assoc. 58, 301 (1963), 236-244. https://doi. org/10.1080/01621459.1963.10500845

[21] Hyewon Kim, MiYoung Lee, and MinJeong Kim. 2014. Effects of Mobile Instant Messaging on Collaborative Learning Processes and Outcomes: The Case of South Korea. Educational Technology \& Society 17, 2 (2014), 31-42.

[22] Neha Kumar, Pallavi Lal, Richard Anderson, Trevor Perrier, Michelle Desmond, Kiersten Israel-Ballard, Vikrant Kumar, Sudip Mahapatra, Anil Mishra, Shreya Agarwal, and Rikin Gandhi. 2015. Projecting Health: Community-Led Video Education for Maternal Health. Proceedings of the Seventh International Conference on Information and Communication Technologies and Development - ICTD '15 (2015), 1-10. https://doi.org/10.1145/2737856.2738023

[23] Shaimaa Lazem and Hussein Aly Jad. 2017. We play we learn: Exploring the value of digital educational games in Rural Egypt. Conference on Human Factors in Computing Systems - Proceedings 2017-May (2017), 2782-2791. https://doi.org/10.1145/3025453.3025593

[24] Ruixue Liu and Changdi Shi. 2016. Investigating Collaborative Learning Effect in Blended Learning Environment by Utilizing Moodle and WeChat. In Lecture Notes in Computer Science (including subseries Lecture Notes in Artificial Intelligence and Lecture Notes in Bioinformatics). Vol. 9757. 3-13. https://doi.org/10.1007/978-3-319-41165-1_1

[25] Michael A. Madaio, Rebecca E. Grinter, and Ellen W. Zegura. 2016. Experiences with MOOCs in a West-African Technology Hub. Proceedings of the Eighth International Conference on Information and Communication Technologies and Development - ICTD '16 (2016), 1-4. https://doi.org/10.1145/2909609.2909633

[26] Carleen Maitland and Eric Obeysekare. 2015. The creation of capital through an ICT-based learning program. Proceedings of the Seventh International Conference on Information and Communication Technologies and Development - ICTD '15 (2015), 1-10. https://doi.org/10.1145/ 2737856.2738024

[27] H. B. Mann and D. R. Whitney. 1947. On a Test of Whether one of Two Random Variables is Stochastically Larger than the Other. The Annals of Mathematical Statistics 18, 1 (1947), 50-60. https://doi.org/10.1214/ aoms/1177730491

[28] Roberto Martinez-Maldonado, Yannis Dimitriadis, Andrew Clayphan, Juan A. Muñoz-Cristóbal, Luis P. Prieto, María Jesús Rodríguez-Triana, and Judy Kay. 2013. Integrating orchestration of ubiquitous and pervasive learning environments. Proceedings of the 25th Australian Computer-Human Interaction Conference on Augmentation, Application, Innovation, Collaboration - OzCHI '13 (2013), 189-192. https: //doi.org/10.1145/2541016.2541076

[29] Maletsabisa Molapo and Gary Marsden. 2013. Software support for creating digital health training materials in the field. Proceedings of the Sixth International Conference on Information and Communication Technologies and Development Full Papers - ICTD '13 - volume 1 (2013), 205-214. https://doi.org/10.1145/2516604.2516632

[30] Concord Newsdesk. 2016. Cameroon: 2016 General Baccalaureate Results Released. Cameroon Concord (Jul 2016). http://cameroon-concord. com/local/cameroon-2016-general-baccalaureate-results-released

[31] Amy Ogan, Vincent Aleven, and C Jones. 2009. Advancing development of intercultural competence through supporting predictions in narrative video. Fournal of Artificial Intelligence in Education 19 (2009), 267-288. http://iospress.metapress.com/index/T53202621973352P.pdf

[32] Kweku Opoku-Agyemang, Bhaumik Shah, and Tapan S. Parikh. 2017. Scaling Up Peer Education with Farmers in India. Proceedings of the
Ninth International Conference on Information and Communication Technologies and Development - ICTD '17 (2017), 1-10. https://doi.org/ 10.1145/3136560.3136567

[33] Niranjan Pai, Pradnya Supe, Shailesh Kore, Y. S. Nandanwar, Aparna Hegde, Edward Cutrell, and William Thies. 2013. Using automated voice calls to improve adherence to iron supplements during pregnancy. Proceedings of the Sixth International Conference on Information and Communication Technologies and Development Full Papers - ICTD '13 volume 1 (2013), 153-163. https://doi.org/10.1145/2516604.2516608

[34] U.S. Pawar, J. Pal, and K. Toyama. 2006. Multiple Mice for Computers in Education in Developing Countries. Information and Communication Technologies and Development (2006), 64-71. https://doi.org/10.1109/ ICTD.2006.301840

[35] Trevor Perrier, Nicola Dell, Brian DeRenzi, Richard Anderson, John Kinuthia, Jennifer Unger, and Grace John-Stewart. 2015. Engaging Pregnant Women in Kenya with a Hybrid Computer-Human SMS Communication System. Proceedings of the 33rd Annual ACM Conference on Human Factors in Computing Systems - CHI '15 (2015), 1429-1438. https://doi.org/10.1145/2702123.2702124

[36] RapidSMS. 2013. About RapidSMS. https://www.rapidsms.org/

[37] Henry L. Roediger and Andrew C. Butler. 2011. The critical role of retrieval practice in long-term retention. Trends in Cognitive Sciences 15, 1 (2011), 20-27. https://doi.org/10.1016/j.tics.2010.09.003

[38] Ridwan Sanjaya and Saranphong Pramsane. 2006. Mobile Education Services Based on SMS and Their Architecture Comparison. Proceedings of third International Conference on eLearning for Knowledge-Based Society (2006), 49.1-49.9. http://ijcim.th.org/SpecialEditions/v14nSP1/ pdf/p49.1-9-fin-61.pdf

[39] Eusebio Scornavacca and Stephen Marshall. 2007. TXT-2-LRN: Improving students' learning experience in the classroom through interactive SMS. Proceedings of the Annual Hawaii International Conference on System Sciences (2007), 1-8. https://doi.org/10.1109/HICSS.2007.579

[40] Simon So. 2016. Mobile instant messaging support for teaching and learning in higher education. Internet and Higher Education 31 (2016), 32-42. https://doi.org/10.1016/j.iheduc.2016.06.001

[41] Piya Sorcar, Benjamin Strauber, Prashant Loyalka, Neha Kumar, and Shelley Goldman. 2017. Sidestepping the Elephant in the Classroom: Using Culturally Localized Technology to Teach Around Taboos. Proceedings of the 2017 CHI Conference on Human Factors in Computing Systems - CHI '17 (2017), 2792-2804. https://doi.org/10.1145/3025453. 3025958

[42] Sasivimon Sukaphat. 2007. Applying of Bulk SMS System to Enhance Educational Communications. Infotech.Monash.Edu.Au (2007), 582586. http://www.infotech.monash.edu.au/research/centres/cdsesr/ apmc/papers-pdf/a276.pdf

[43] Ying Tang and Khe Foon Hew. 2017. Is mobile instant messaging (MIM) useful in education? Examining its technological, pedagogical, and social affordances. Educational Research Review 21 (2017), 85-104. https://doi.org/10.1016/j.edurev.2017.05.001

[44] Samaneh Tarighat and Samaneh Khodabakhsh. 2016. Mobile-Assisted Language Assessment: Assessing speaking. Computers in Human Behavior 64 (nov 2016), 409-413. https://doi.org/10.1016/j.chb.2016. 07.014

[45] Heather Underwood, Clint Tseng, Charlotte Robinson, Sunil Garg, Meera Lakshmanan, Richard Anderson, and Joyojeet Pal. [n. d.]. MultiMath : Using Numeric Keypads to Utilize Limited Computer Resources for Education. ([n. d.]), 3-5.

[46] Silje C Wangberg, Eirik Årsand, and Niklas Andersson. 2006. Diabetes education via mobile text messaging. Fournal of Telemedicine and Telecare 12, 1_suppl (2006), 55-56. https://doi.org/10.1258/ 135763306777978515 
[47] Nikolas Wolfe, Juneki Hong, Agha Ali Raza, Bhiksha Raj, and Roni Rosenfeld. 2015. Rapid Development of Public Health Education Systems in Low-Literacy Multilingual Environments : Combating Ebola Through Voice Messaging. November (2015), 131-136. https: //doi.org/10.13140/RG.2.1.1885.6405 